\title{
Análisis de la producción científica sobre Gobierno Corporativo a través de ISI Web of Science
}

\author{
Alicia Richart-Ramón*, Mónica Martínez-Blasco* y Josep García-Blandón*
}

\begin{abstract}
Resumen: El buen gobierno de las empresas constituye en la actualidad uno de los principales temas de investigación en el ámbito académico de economía y empresa. El interés del tema no es únicamente académico, si tenemos en cuenta el elevado número de códigos de buen gobierno promulgados durante los últimos años, o la responsabilidad atribuida a las cuestiones de gobierno empresarial en la actual crisis financiera. Este trabajo ha analizado 2.147 publicaciones sobre buen gobierno empresarial incluidas en la base de datos ISI Web of Science y sus correspondientes 79.635 referencias con el objetivo principal de identificar las pautas sobre las que se basa la investigación. En particular, hemos identificado las publicaciones y los autores más relevantes, los más productivos, las revistas en que se han publicado las investigaciones y las aproximaciones utilizadas para abordar el tema.
\end{abstract}

Palabras clave: producción científica, gobierno corporativo.

\section{Research in Corporate Governance: An analysis through ISI Web of Science}

\begin{abstract}
Corporate governance constitutes nowadays one of the main topics of research in the fields of economics and business. The importance of the subject is not merely academic, if we consider the large number of codes of corporate governance promulgated in recent years, or the importance of corporate governance issues in the current international financial crisis. This paper has analyzed 2,147 publications on corporate governance included in ISI Web of Science, and their corresponding 79,635 citations, with the aim of identifying the prevailing research patterns in the field. In particular, we have traced the most significant publications and authors, the most productive authors, the journals in which they have published, and the approaches used to carry out the investigation.
\end{abstract}

Keywords: Scholarly production, corporate governance.

\section{Introducción}

La investigación de la relación entre propiedad y dirección en la empresa constituye, desde principios de los años 30, una de las principales líneas de interés en el ámbito de economía y empresa. En un trabajo pionero, Berle y Means

* Departamento de Economía y Finanzas. Facultad de Economía IQS. Universidad Ramón Llull. Barcelona. Correo-e: alicia.richart@iqs.edu; monica.martinez@iqs.edu; josep.garcia@iqs.edu.

Recibido: 08-04-2010; 2. ${ }^{a}$ versión: 03-06-2010; 3. . versión: 15-06-2010; aceptado: 17-06-2010. 
(1932) afirman que si la propiedad de las grandes corporaciones es dispersa, las acciones que sus propietarios pueden ejercer sobre las decisiones que toman los directivos es limitada. Estas circunstancias generan tensiones entre directivos y propietarios de las empresas que se recoge como una línea de investigación con entidad propia y que se denomina teoría de la agencia. Jensen y Meckling (1976) describen las distintas formas que podían adoptar los problemas de agencia y que en definitiva provocan que los insiders (accionistas mayoritarios y directivos, principalmente) de las compañías se beneficien a costa de los outsiders (accionistas minoritarios y otros grupos de interés). Ya entonces quedó clara la necesidad de que los accionistas minoritarios de las empresas tuvieran recursos para poder proteger sus intereses y se planteó la elaboración de normativas legales como una posible solución.

Tras los múltiples escándalos que destaparon fraudes y otros tipos de abusos provocados por altos cargos de empresas tanto americanas como europeas en la década de los noventa (Enron, WorldCom, Parmalat, entre otros), diversas instituciones de distintos países empezaron a promover normativas, recomendaciones y códigos de buen gobierno. El objetivo de todos ellos era proteger a los accionistas del exceso de poder de los directivos y asegurar que éstos cumplieran con su cometido de gestionar la empresa tanto para sus accionistas como para todo aquel que tuviera una relación con la compañía. La institucionalización de la forma en que los directivos debían relacionarse con su entorno dio impulso a una línea de investigación que hasta aquel entonces se encontraba circunscrita al ámbito de la teoría de la agencia, el análisis del gobierno corporativo de las empresas, y le concedió entidad propia. De acuerdo con López y Pereira (2006), si bien los primeros códigos de buen gobierno datan de finales de los años 80, a finales de los años 90 se habían publicado un total de 72 códigos. De los 33 códigos correspondientes a países de la Unión Europea, 25 se habían promulgado a partir de 1997. A pesar del esfuerzo realizado para garantizar el buen gobierno empresarial, el 9 de Diciembre del pasado año 2009, el responsable del Tesoro británico, Alistair Darling, anunció la aplicación de un impuesto excepcional del 50\% para los bonus a los altos ejecutivos de la banca que superasen las 25.000 libras, poniendo de manifiesto la actualidad del problema.

El término gobierno corporativo se venía utilizando en ciencias sociales desde mediados de los años setenta pero no es hasta finales de los noventa cuando el término adquiere relevancia; Farinha (2003) apunta cuatro razones por las que ha aumentado el debate y el interés por el gobierno corporativo desde finales de los años noventa: por un lado, diversos autores pusieron en duda la eficacia de los mecanismos que controlan el gobierno de las empresas, citando algunos autores de referencia como Jensen (1993), Miller (1997) o Porter (1997); en segundo lugar, los escándalos financieros, los elevados salarios de los ejecutivos, hechos además públicos por la prensa, así como la adopción de medidas de bloqueo por parte de los directivos. La tercera razón es precisamente la elaboración de legislaciones para limitar el papel de los directivos en las medidas 
antibloqueo que adoptan. Por último, considera que el debate abierto para comparar las distintas estructuras de gobierno corporativo entre Estados Unidos, Alemania y Japón (Shleifer y Vishny, 1997) y el número de iniciativas tomadas por los mercados financieros y otras autoridades en la elaboración de códigos y recomendaciones de gobernabilidad, hicieron visibles los temas relacionados con el buen gobierno de las empresas Farinha (2003).

Distintos autores han definido el concepto gobierno corporativo desde varios puntos de vista. Sin ánimo de ser exhaustivos, se han escogido las definiciones o justificaciones que, en nuestra opinión, resultan más acertadas. Hart (1995) entiende que «los problemas de gobierno aparecen en una organización cuando cualquiera de las siguientes condiciones están presentes: la primera, si existe un problema de agencia o conflicto de interés, incluyendo a miembros de la organización como propietarios, directivos, trabajadores o consumidores y, segunda, los costes de transacción para solucionar el conflicto son tales que el problema de agencia no se puede resolver a través de un contrato" (p. 678). Shleifer y Vishny (1997) definen el gobierno corporativo como "todo aquello que permite a los suministradores de recursos financieros de las empresas asegurarse un rendimiento a su inversión" (p. 737). Zingales (1998) plantea que "la propiedad, la estructura de capital, los esquemas de incentivos, las adquisiciones hostiles, el consejo de administración, la presión de los inversores institucionales, la competencia del mercado de un producto, la competencia en el mercado laboral y la estructura organizativa, entre otros, son conceptos o instituciones que afectan el proceso de distribución de rentas (p. 4) por lo que define gobierno corporativo como «el conjunto de restricciones que definen el procedimiento de reparto de las rentas generadas por una empresa". Un gobierno corporativo eficiente implica mecanismos que aseguren que los directivos respetarán los derechos y los intereses de los accionistas y asimismo darán cuenta de que sus decisiones son responsables y comprometidas con la protección, la generación y la distribución de la riqueza generada en la firma [Filatotchev y Boyd, 2009]. Tras más de una década de los primeros escándalos que motivaron la elaboración de legislaciones y códigos de buen gobierno, el debate sigue estando si cabe de mayor actualidad. La honestidad de las prácticas contables así como los límites de actuación de los directivos están de nuevo en entredicho después de que el sistema financiero mundial haya entrado en crisis, en buena medida por excesos contables y financieros. Estos hechos han reabierto el debate entorno a cómo reforzar los mecanismos que permiten un buen gobierno corporativo dado que una de las causas de la actual crisis ha sido precisamente la debilidad del gobierno corporativo de las empresas.

Tal y como apuntan Ahrens y otros (2009): "Yet despite this enourmous volumen of research [hablando de la producción científica en gobierno corporativo entre 2007 y 2009] we still know very little about corporate governance" (p. 2). Según Correa y otros (2009), la investigación en arquitectura financiera internacional, donde se incluye la investigación en gobierno corporativo, es la segunda línea de investigación con más artículos publicados en Economía Financiera en- 
tre 1995 y 2006. De acuerdo con los autores, "El creciente interés por parte de las empresas, los gobiernos y la opinión pública, ha hecho que esta línea de investigación adquiera relevancia, pasando por delante de la política de dividendos [...]" (p. 89).

A pesar de la ingente investigación realizada y del interés creciente del tema, existen pocos antecedentes de trabajos que hayan analizado la producción científica sobre gobierno corporativo. Merece la pena destacar toda una serie de trabajos que han puesto el énfasis en recopilar y analizar el avance de la investigación en el tema que nos atañe. Nos parece especialmente interesante destacar entre ellos el publicado por Shleifer y Vishny (1997) en el que los autores, con un claro enfoque hacia los problemas de agencia, resumen las principales líneas $\mathrm{y}$ autores que trabajan en gobierno corporativo y ponen orden a lo que ellos llaman "unresolved puzzles in the analysis of corporate governance" (p. 5). En esta línea, Farinha (2003) publica una compilación de la literatura publicada sobre gobierno corporativo con el objetivo de establecer los puntos en los que los investigadores han alcanzado un mayor consenso y aquéllos en los que éste no ha sido posible. Becht y otros (2003) revisan de forma extensa la investigación teórica y empírica desarrollada sobre los mecanismos básicos de control en el gobierno corporativo en el ámbito internacional, clasificando a los autores a partir del tema de investigación.

Recientemente, Durisin y Puzone (2009) analizan mil artículos en los que aparecía el término corporate governance. Los autores escogen para el análisis de la producción científica la revista de referencia Corporate Governance: An International Review desde 1993 hasta el 2007, así como otras 15 revistas académicas consideradas como líderes en este campo con el objetivo de identificar los trabajos más influyentes en el área. También en 2009, Filatotchev y Boyd (2009) elaboran un editorial para Corporate Governance: An International Review donde analizaron ocho artículos que ellos consideran principales en gobierno corporativo y plantean futuras líneas de investigación.

Otros trabajos analizan y recopilan la literatura existente desde un punto de vista nacional o de región: Asia [Claessen y Fan, 2003], Europa del Este [Walsh y Whelan, 2001], Alemania [Prigge, 1998], Unión Soviética [Estrin y Writght, 1999] y Canadá [Lindsay y otros, 1996].

La principal finalidad de este trabajo es ofrecer una visión global del estado de la investigación sobre gobierno corporativo con el objetivo de identificar las bases de la investigación científica, su evolución a lo largo de los años así como de sus intereses, a efectos de facilitar su investigación futura. Para ello, en este trabajo se analiza la producción científica en el ámbito del gobierno corporativo a través de la base de datos ISI Web of Science (ISI WoS), con el objetivo de determinar los autores de referencia, las revistas más importantes donde se publican los resultados de la investigación así como los principales temas de investigación seguidos en este área. El presente trabajo extiende la literatura ya existente sobre el tema con dos aportaciones básicas: por un lado, se utiliza la base de datos de referencia en investigación científica para realizar el análisis, por lo 
que el número de referencias estudiadas es de mayor alcance y amplitud que en trabajos anteriores. Por otro lado, el período temporal estudiado es también más amplio que el analizado en los trabajos previos sobre el tema.

En el siguiente apartado se presenta la metodología empleada así como los datos analizados y en el apartado 3 se presentan los resultados obtenidos del estudio. Finalmente, en el apartado 4 se exponen las principales conclusiones.

\section{Metodología}

El análisis planteado se ha realizado a partir de una búsqueda bibliográfica en la base de datos ISI Web of Science (ISI WoS), que incluye Science Citation Index Expanded (SCIE), Social Sciences Citation Index (SSCI) y Arts \& Humanities Citation Index (AHCI), y que suministra información referente a título, autores, palabras clave, resúmenes y referencias citadas, entre otros detalles, de un total aproximado de diez mil revistas científicas de alto impacto. El mayor alcance de nuestra investigación al utilizar ISI Web of Knowledge queda justificado en distintos trabajos como por ejemplo en Reyes y otros (2006), Martin y Irvine (1983), Instituto de Estudios Documentales sobre Ciencia y Tecnología (2009), Bordons y otros (1996), Bordons y Gómez-Caridad (1997), Jiménez y Moya (2003) y Moya y otros (2005).

La búsqueda se ha organizado por temática, se ha introducido el término equivalente en inglés a gobierno corporativo por lo que se escribieron las palabras corporate governance en la línea de TOPIC de la base de datos de ISI WoS. Esta primera búsqueda se refinó excluyendo cualquier tipo de documentación que no fueran artículos y escogiendo las áreas de conocimiento correspondientes a "Business", "Economics", "Management", "Business, Finance", "Law" y "Ethics", que representan el 85\% de la muestra. No se limitó ninguna fecha de inicio en la búsqueda aunque sí la fecha más actual. Se escogió desde el inicio temporal de registro en la base de datos hasta 2008 para poder cerrar un año completo de publicaciones y poder hacer el análisis anual. Si se hubiera incluido 2009, al no tener certeza de cuándo se acabarían de actualizar las publicaciones, se estaría trabajando con datos sesgados. El resultado de la búsqueda realizada bajo las anteriores condiciones proporcionó un total de 2.147 artículos, estando el primero de ellos registrado en 1977 (Estes, 1977) y los últimos de acuerdo con lo anteriormente apuntado en 2008.

Los datos se descargaron a través de la Web y se exportaron desde la página de resultados seleccionando todos los campos de exportación que ofrece la herramienta, incluido el registro completo y las referencias citadas, y guardados en archivos delimitados por tabuladores de 500 en 500 referencias. Una vez extraída toda esta información a una única hoja de cálculo, se procesaron los datos de tal forma que se pudiera analizar la evolución de la actividad científica y que se pudiera contar con el volumen de producción científica por año, los artículos más relevantes por números de citas y su evolución temporal, los autores más 
destacados y las revistas con mayor volumen de publicación, así como de las citas recibidas durante el período de estudio. También nos interesó la evolución que seguían las palabras clave que resumen los temas principales tratados en los contenidos de los artículos con el objetivo de poder analizar cómo evolucionan en el tiempo los intereses científicos en este área. La metodología seguida en este punto ha sido la siguiente:

Se han extraído todas las palabras claves de artículos del período de disponibilidad, en este caso desde 1990 hasta 2008.

Se han ordenado y agrupado por similitud.

Se ha eliminado la palabra clave "gobierno corporativo" por ser el tema central de la investigación.

Se han dividido en dos grupos: palabras clave relacionadas con temas intrínsecos al gobierno corporativo y palabras claves relacionadas con el ámbito económico-financiero en general.

\section{Resultados relativos a las publicaciones en el ámbito del gobierno corporativo}

En esta sección se analiza la evolución temporal del número de publicaciones, la productividad de los autores así como los autores principales en la materia, la productividad de las revistas y las revistas más influyentes. Por último, también se estudia la evolución de las palabras clave sobre la investigación de gobierno corporativo.

Un análisis general de los datos obtenidos muestra que los artículos publicados en ISI WoS sobre gobierno corporativo fundamentalmente están escritos en inglés $(97,67 \%)$, seguidos por el francés $(0,51 \%)$ y el alemán $(0,31 \%)$ de forma casi anecdótica. Respecto a los países desde los que se publica, encabezan el listado los países anglosajones con Estados Unidos como productor principal (1.165 artículos), seguido por Reino Unido (298 artículos) y Canadá (130 artículos). El resto de países se encuentran en niveles de producción mucho más bajos.

Las principales subáreas donde se integran este tipo de artículos según la clasificación de ISI son el área de "Business" (33,34\%), el área de "Economics" (30,32\%), seguidos por "Management" (29,15\%) y "Business, Finance" (27,85\%). Los artículos que abordan el tema del gobierno corporativo desde el punto de vista del Derecho $(16,62 \%)$ y la Ética $(5,44 \%)$ son los que representan un porcentaje menor (estos porcentajes no se deben tomar como absolutos ya que un artículo puede estar clasificado en varias áreas de conocimiento a la vez).

A nivel de producción institucional, lidera el volumen de artículos publicados Harvard University, a la que le sigue ya con una cierta distancia Columbia University y la University of Pennsylvania. Las principales revistas utilizadas para divulgar los resultados de la investigación sobre gobierno corporativo son Corporate Governance: An International Review y el Journal of Financial Economics. 
TABLA I

Número de publicaciones por institución

\begin{tabular}{l|c|c}
\hline \multicolumn{1}{c|}{ Institución } & $\begin{array}{c}\text { Número } \\
\text { de publicaciones }\end{array}$ & $\begin{array}{c}\text { Porcentaje que representan } \\
\text { sobre la muestra }\end{array}$ \\
\hline Harvard University & 85 & 3,95 \\
\hline Columbia University & 41 & 1,91 \\
\hline University of Pennsylvania & 41 & 1,91 \\
\hline University of Nottingham & 39 & 1,81 \\
\hline New York University & 38 & 1,77 \\
\hline University of Chicago & 37 & 1,72 \\
\hline University of Michigan & 35 & 1,63 \\
\hline University of Cambridge & 31 & 1,44 \\
\hline University of Texas & 29 & 1,35 \\
\hline Indiana University & 27 & 1,26 \\
\hline Stanford University & 27 & 1,26 \\
\hline Texas AEM University & 26 & 1,21 \\
\hline Total & 456 & 21,20 \\
\hline
\end{tabular}

Fuente: ISI WoS.

\subsection{Evolución del número de publicaciones}

La evolución del volumen de artículos publicados sobre gobierno corporativo se muestra en la figura 1. Se puede comprobar como el número de artículos publicados sigue una tendencia creciente después de una fase de estancamiento que abarca desde la primera publicación de finales de los setenta y hasta principios de los años noventa. Los años 1992 y 1993 se pueden considerar años en los que se produce un impulso en cuanto al número de referencias publicadas ya que se duplica la producción de artículos pero es a partir del año 1996 donde el número de publicaciones ya es creciente año tras año. De esta forma, entre el año 2000 y el 2008 se publicó alrededor del 80\% de la literatura existente (1.711 artículos sobre un total de 2.147) en nuestra muestra. Quizás incluso más interesante que el número absoluto de artículos publicados en el período sea que el máximo número de publicaciones se alcanza en el último año del período investigado (con 346 publicaciones), hecho que muestra la actualidad y el interés de este área de investigación.

De hecho, el número de artículos publicados por año crece exponencialmente según la Ley de crecimiento exponencial de Price. La ecuación que modeliza este crecimiento se muestra superpuesta en la figura 1. De las constantes de esta función se han extraído la tasa de crecimiento anual y el tiempo de duplicación 
FIGURA 1

Número de artículos publicados

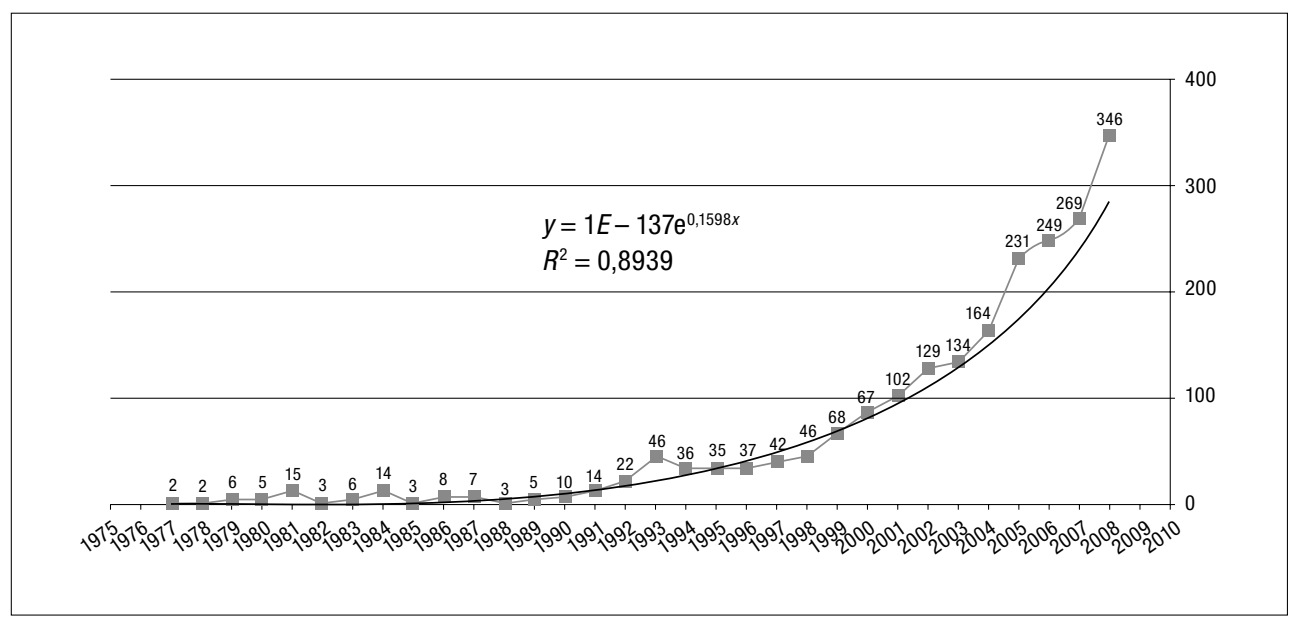

Fuente: elaboración propia a partir de los datos de WoS.

de la producción científica. En nuestro caso la tasa de crecimiento anual es de $17,33 \%$ y el tiempo de duplicación de 4,34 años.

Dado que ninguna disciplina científica crece indefinidamente de forma exponencial pura, ésta debe alcanzar un momento en el cual el proceso se debilita para detenerse antes de llegar al absurdo. El proceso debe regirse por una curva logística limitada por un techo, o línea de saturación, después del cual el crecimiento no puede continuar en su manera acostumbrada. En las primeras etapas, el crecimiento se asemeja al exponencial puro, para posteriormente ir frenándose y producir una inflexión en la que el crecimiento disminuye de forma simétrica hasta acercarse asintóticamente al límite de saturación. Es lo que se conoce como Ley de crecimiento logístico o segunda Ley de Price.

\subsection{Autores}

Esta sección se ha estructurado en tres apartados. En primer lugar, se ha realizado la distribución de los autores de las 2.147 publicaciones analizadas en función del número de artículos publicados. Esta clasificación nos permitirá determinar el grado de especialización de los investigadores sobre gobierno corporativo. A continuación, se han seleccionado los artículos más citados, sus autores y la evolución temporal del número de citas recibidas, a efectos de identificar cuáles son las publicaciones y autores de referencia en gobierno corporativo y cómo ha evolucionado en el tiempo la importancia de estos trabajos de referencia. Este apartado finaliza con la identificación de los autores más productivos. 


\subsubsection{Productividad de los autores}

Si se analiza la tipología de autores por número de artículos producidos (tabla II) se puede constatar que de los 3.004 autores que han firmado los 2.147 artículos de la muestra, prácticamente el $80 \%$ son autores ocasionales, publicando un solo artículo.

Dentro de los denominados grandes autores, con 10 o más documentos publicados, encontramos a 7 autores que representan el 0,23\% mientras que como autores medianos, entre 2 y 9 documentos, coexistirían 610 autores que representan casi el 20,31\%.

TABLA II

Productividad de los autores

\begin{tabular}{|c|c|c|c|}
\hline Tipologías de autores & $\begin{array}{l}\mathbf{N}^{\circ} \text { artículos } \\
\text { por autor }\end{array}$ & $\mathbf{N}^{\mathrm{o}}$ autores & $\begin{array}{l}\text { Porcentaje que representan } \\
\text { sobre la muestra }\end{array}$ \\
\hline \multirow{5}{*}{ Grandes autores } & 23 & 1 & \multirow{5}{*}{0,23} \\
\hline & 14 & 1 & \\
\hline & 12 & 2 & \\
\hline & 11 & 1 & \\
\hline & 10 & 2 & \\
\hline \multirow{8}{*}{ Autores medianos } & 9 & 5 & \multirow{8}{*}{20,31} \\
\hline & 8 & 9 & \\
\hline & 7 & 4 & \\
\hline & 6 & 17 & \\
\hline & 5 & 31 & \\
\hline & 4 & 45 & \\
\hline & 3 & 116 & \\
\hline & 2 & 383 & \\
\hline Autores ocasionales & 1 & 2.387 & 79,46 \\
\hline \multicolumn{2}{|l|}{ Número total de autores } & 3.004 & 100,00 \\
\hline
\end{tabular}

Fuente: elaboración propia a partir de los datos de WoS.

Al disponer en un gráfico el número de publicaciones por autor, se observa que la muestra sigue una distribución clásica de la Ley cuadrática inversa de la productividad de los autores de Lotka (figura 2). Ésta determina que partiendo del número de autores con un solo trabajo en un tema determinado es posible predecir el número de autores con n trabajos mediante el cociente entre el número de autores de una firma y $n^{2}$ (López, 1996). 
FIGURA 2

Distribución de los autores según su productividad

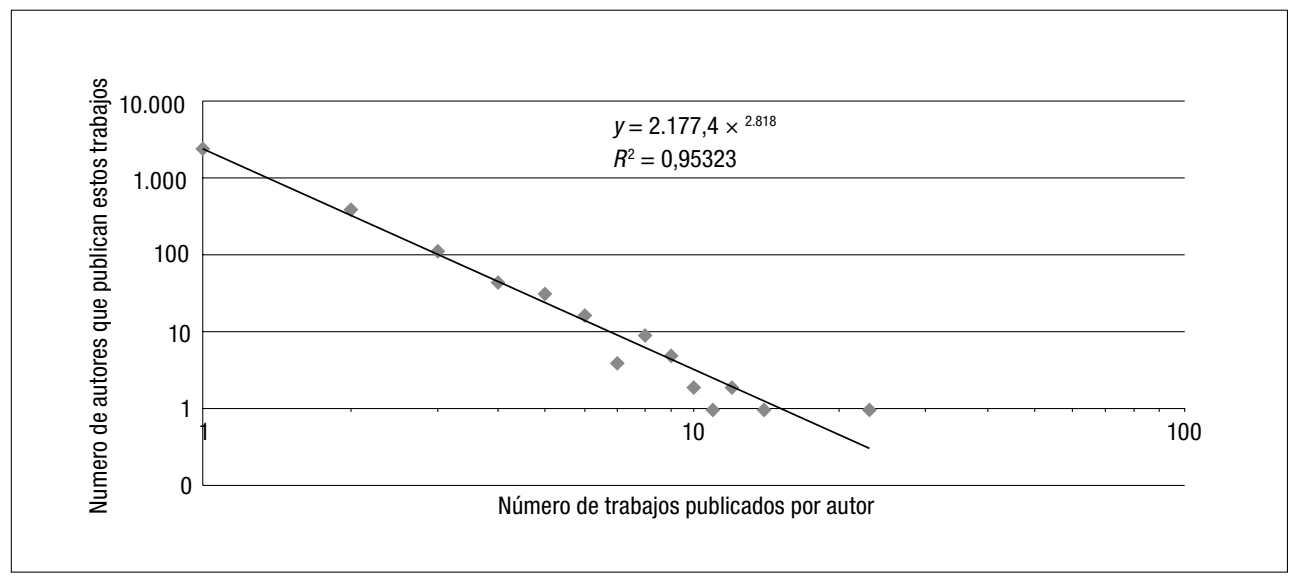

Fuente: elaboración propia a partir de los datos de WoS.

\subsubsection{Autores más relevantes}

A efectos de identificar a los autores más relevantes, se ha utilizado el criterio habitual para determinar el impacto de una publicación como es el "número de citas". Se entiende que la citación de un documento refleja interés y conocimiento en la articulación de un nuevo concepto, a pesar de diversos factores que puedan influir (Bordons y Zulueta 1999). En la tabla III se presentan los artículos más citados, de tal forma que se puedan identificar los fundamentos sobre los que se está construyendo las líneas de investigación sobre gobierno corporativo. De dichos artículos se ha detallado el nombre de los autores, el año de publicación, el título, la revista y el número de veces que éste aparece citado de forma absoluta y en promedio anual en las referencias. Rafael La Porta, Florencio López-de-Silanes, Andrei Shleifer y Robert Vishny (1997) son los autores más citados en los trabajos de otros investigadores, contabilizando 2.065 citas repartidas entre sus cuatro artículos que aparecen en el ranking. Entre todos ellos destaca el trabajo seminal de La Porta y otros (1998) citado en 1.019 ocasiones desde su publicación. Siguiendo en orden de relevancia a La Porta y otros $(1998,1999)$ se encuentran los artículos de Yermarck (1996) y Gompers y otros (2003). De los artículos más citados se puede deducir que los intereses de los investigadores se centran, en primer lugar, en ampliar las investigaciones seguidas por La Porta y otros (1998, 1999, 2000, 2002). Estos autores concentran sus esfuerzos en investigar los mecanismos, principalmente legales, que mejor protegen a los accionistas de las empresas y su relación con el tamaño de la compañía, la estructura de propiedad y la rentabilidad con un enfoque global [Durisin y Puzone, 2009]. Por otro lado, destacan también los artículos que relacionan el valor de la empresa con su gobierno corporativo y por tanto trabajos que miden si existe una recompensa por parte del mercado a 
un buen gobierno corporativo, como es el caso de Gompers y otros (2003), trabajos que relacionan el tamaño del consejo de administración con el valor de la empresa [Yermarck, 1996] o la compensación de los ejecutivos [Davis, 1991].

A pesar de no encontrarse registrado en la tabla III por no tratarse de un artículo, merece la pena destacar el hecho de que el libro de Adolf A. Berle y Gardiner C. Means "The Modern Corporation and Private Property" aparecería en la sexta posición de la tabla de referencias más citadas ya que el análisis bibliográfico muestra que se ha hecho referencia al libro en un total de 206 ocasiones. Este libro es considerado el origen de las finanzas modernas y punto de partida para la investigación sobre finanzas corporativas y gobierno corporativo.

Respecto a la evolución en el número de citas en el período considerado (figura 3), el artículo de La Porta y otros (1998) sigue una tendencia creciente año tras año en el número de citas, por lo que las líneas de investigación abiertas por estos autores parecen ser las que despiertan un mayor interés por los investigadores. La misma tendencia siguen el resto de artículos, exceptuando los de Dodd y Warner (1983), Williamson (1988) y Davis (1991) que corresponden también a los artículos más antiguos.

\section{FIGURA 3}

Citas recibidas por año

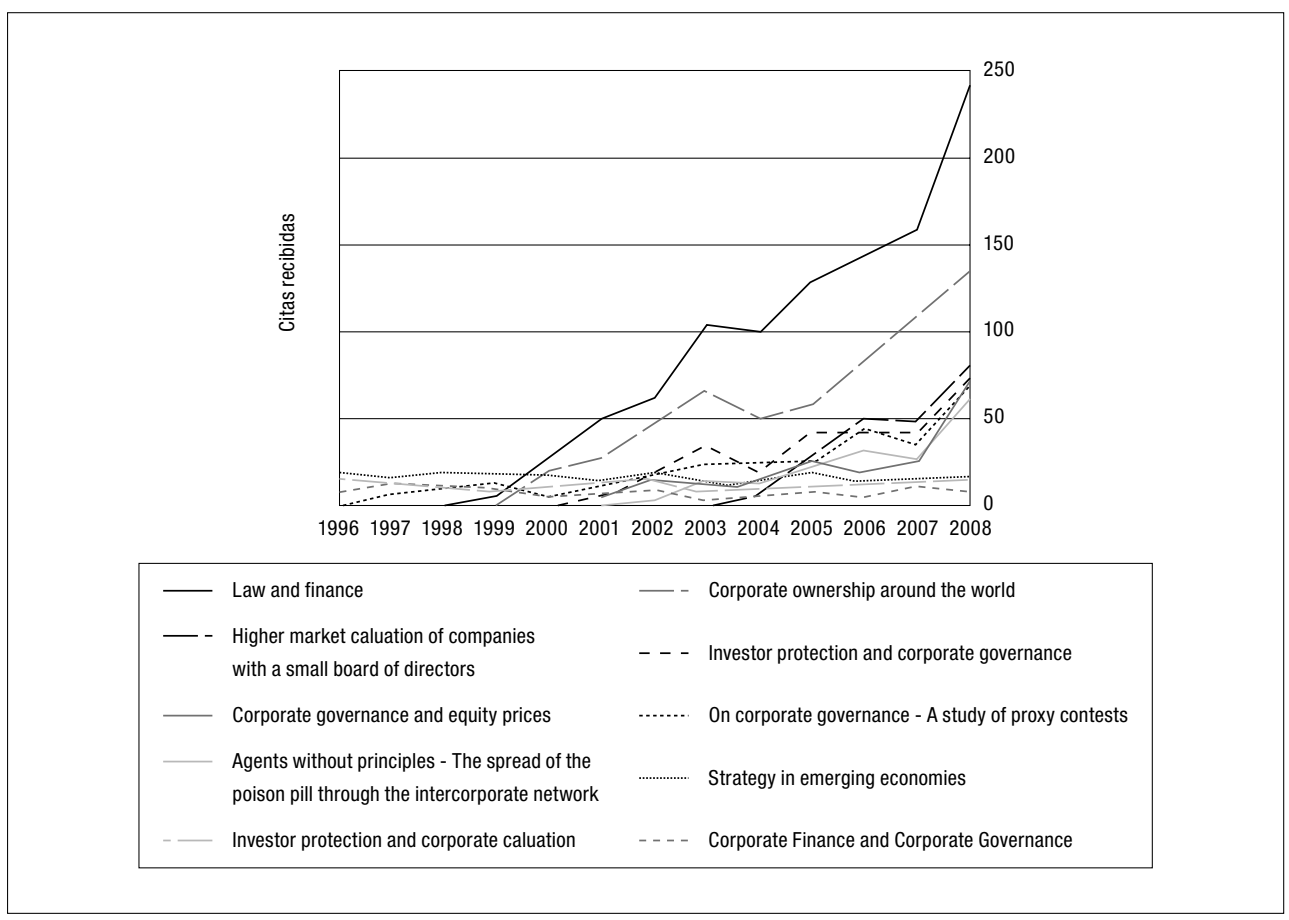

Fuente: elaboración propia a partir de los datos de ISI WoS. 


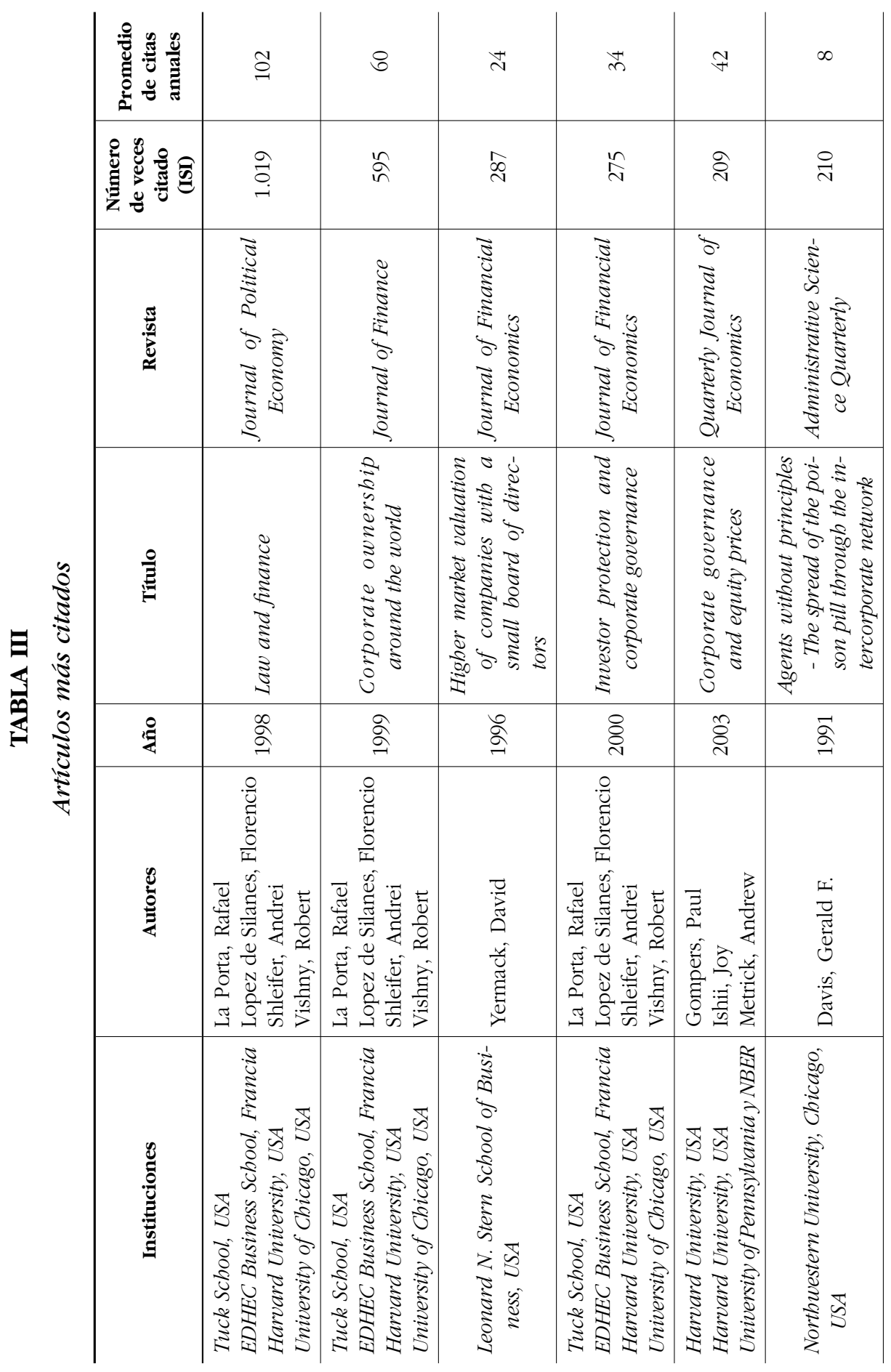




\begin{tabular}{|c|c|c|c|c|}
\hline 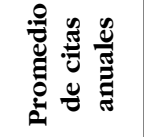 & $\underset{\sim}{\circ}$ & $\approx$ & $\stackrel{n}{n}$ & $\stackrel{\sim}{\sim}$ \\
\hline 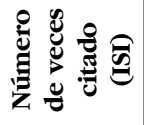 & $\stackrel{10}{\sim}$ & $\stackrel{゚}{\stackrel{2}{2}}$ & 古 & $\stackrel{\vec{\sigma}}{\circ}$ \\
\hline 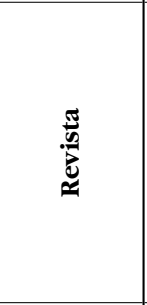 & 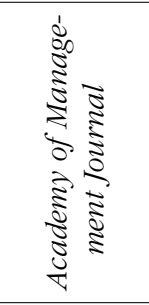 & 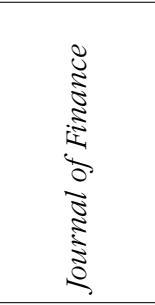 & 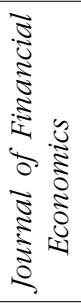 & 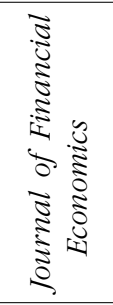 \\
\hline 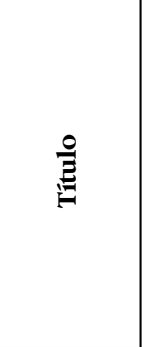 & 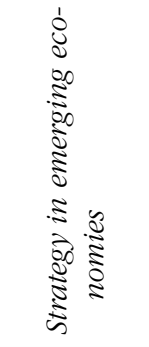 & 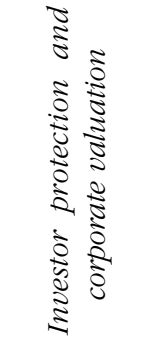 & 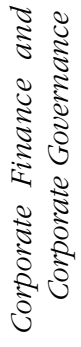 & 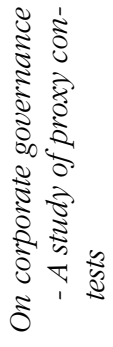 \\
\hline 安 & @্ণ & ণิ & $\begin{array}{l}\infty \\
\stackrel{0}{\rightleftharpoons}\end{array}$ & $\begin{array}{l}\stackrel{\infty}{=} \\
\stackrel{2}{2}\end{array}$ \\
\hline 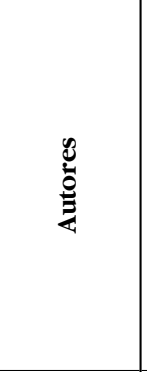 & 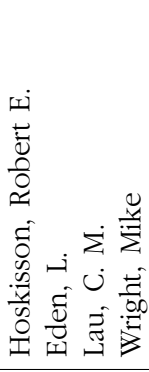 & 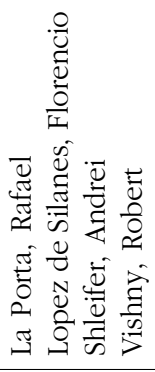 & 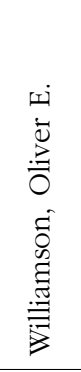 & 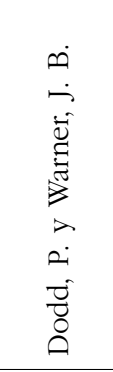 \\
\hline 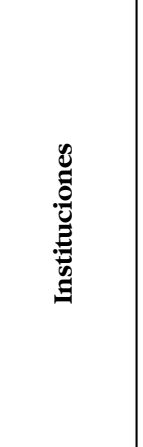 & 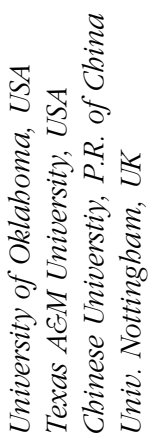 & 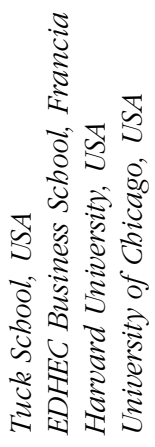 & 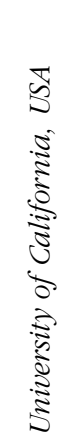 & 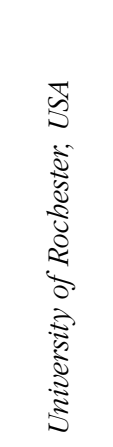 \\
\hline
\end{tabular}




\subsubsection{Autores más productivos}

Además de los autores más relevantes por número de citas, se ha realizado una búsqueda de los autores con mayor volumen de publicación en el período de análisis. El resultado se muestra en la tabla IV. Como puede observarse, entre los autores más citados únicamente Wright figura también entre los que cuentan con un mayor número de publicaciones. De la comparación de las tablas III y IV podemos concluir que no siempre el trabajo de los autores más productivos es el que obtiene más relevancia posteriormente.

\section{TABLA IV}

Autores con mayor número de publicaciones

\begin{tabular}{l|c|l}
\hline \multicolumn{1}{c|}{ Autor } & $\begin{array}{c}\text { Número de } \\
\text { publicaciones }\end{array}$ & \multicolumn{1}{c}{ Institución } \\
\hline Wright, Mike & 23 & University of Nottingham, UK. \\
\hline Filatotchev, Igor & 14 & City University London, UK. \\
\hline Buck, Trevor & 12 & University Loughborough, UK. \\
\hline Dalton, Dan R. & 12 & Indiana University, USA. \\
\hline Thompson, Steve & 11 & University of Nottingham, UK. \\
\hline Bebchuk, Lucian A. & 10 & Harvard University, School of Law, USA. \\
\hline Gilson, Ronald J. & 10 & Stanford Law School, USA. \\
\hline Bainbridge, Stephen M. & 9 & University of California Los Angeles, School of Law, USA. \\
\hline Coffee, John C. & 9 & Columbia University, School of Law, USA. \\
\hline Hoskisson, Robert E. & 9 & Arizona State University, USA. \\
\hline Kang, Jung-Koo & 9 & Northwestern University, USA. \\
\hline Zajac, Edward J. & 137 & \\
\hline Total & 9 & \\
\hline
\end{tabular}

\subsection{Revistas}

El análisis de las revistas científicas se ha desarrollado utilizando un triple enfoque: en qué revistas se han publicado los trabajos más relevantes y qué revistas aparecen como las más referenciadas por estos artículos, qué revistas aparecen como las más referenciadas en el conjunto de las publicaciones analizadas y finalmente qué revistas concentran un mayor porcentaje de los artículos publicados. La utilización de esta aproximación nos permitirá delimitar el rol que ocupa cada revista en la difusión de los resultados de la investigación sobre gobierno corporativo. 


\subsubsection{Análisis de las revistas donde se publican los artículos más relevantes}

De los artículos más relevantes, dado que son los más citados, se han analizado las revistas elegidas por estos autores para publicar su trabajo. Tal y como se puede apreciar en la tabla III, el Journal of Financial Economics y el Journal of Finance, con 4 y 2 artículos publicados respectivamente, son las revistas que presentan una concentración más alta de publicación de artículos más citados con un $60 \%$ entre las dos. El resto de revistas registran un artículo cada una de ellas.

Por lo que respecta al número de citas que reciben las revistas donde se han publicado estos artículos el Journal of Political Economy $(47,46 \%)$ es la revista que más se ha utilizado como referencia. Este hecho se explica en buena medida dado que el artículo de La Porta y otros (1998), el artículo más citado, fue publicado en dicha revista. A continuación se encuentra el Journal of Financial Economics $(38,10 \%)$ con 4 artículos citados 818 veces y el Journal of Finance $(35,91 \%)$ con 2 artículos citados 771 veces. A estas tres revistas le siguen ya a bastante distancia el Administrative Science Quarterly (9,78\%), el Quarterly Journal of Economics (9,73\%) y el Academy of Management Journal (8,62\%) (estos porcentajes no pueden ser tomados en valor absoluto ya que al existir la posibilidad de que un artículo cite a su vez a varios la suma de los porcentajes de las revistas no es cien).

\subsubsection{Revistas más citadas}

Para determinar las revistas con una mayor influencia en esta disciplina se han analizado las revistas donde se han publicado los artículos referenciados en la bibliografía de 1.611 artículos, procesándose un total de 79.635 citas. La muestra inicial de 2.147 trabajos se ha visto reducida ya que la base de datos solo ofrece información bibliográfica extraíble de los artículos publicados a partir de 1996.

En la figura 4 se muestran las 16 revistas que han recibido más citas registrando en total 22.465, o lo que es lo mismo, el 28,20\% del total de citas analizadas. Tal y como se puede comprobar, el Journal of Financial Economics es la revista de referencia para sustentar la base teórica que soporta la investigación sobre gobierno corporativo seguida del Journal of Finance. Investigaciones previas han determinado que estas revistas científicas son las más referenciadas en la investigación en finanzas [entre otros, Borockhovich y otros, 1994 y Chan y otros, 2000]. A pesar de que el gobierno corporativo no es una cuestión específicamente financiera, sino que abarca distintos campos como la economía, la administración de empresas o la ética, este resultado mostraría como de todos los enfoques posibles, los artículos en los que se fundamenta la investigación sobre gobierno corporativo tienen un predominante enfoque financiero. A continuación, si bien a gran distancia de las anteriores, se encuentran dos de las revistas más influyentes circunscritas al área de dirección de empresas como son el Academy of Management Journal y el Strategic Management Journal. 
FIGURA 4

Revistas más citadas

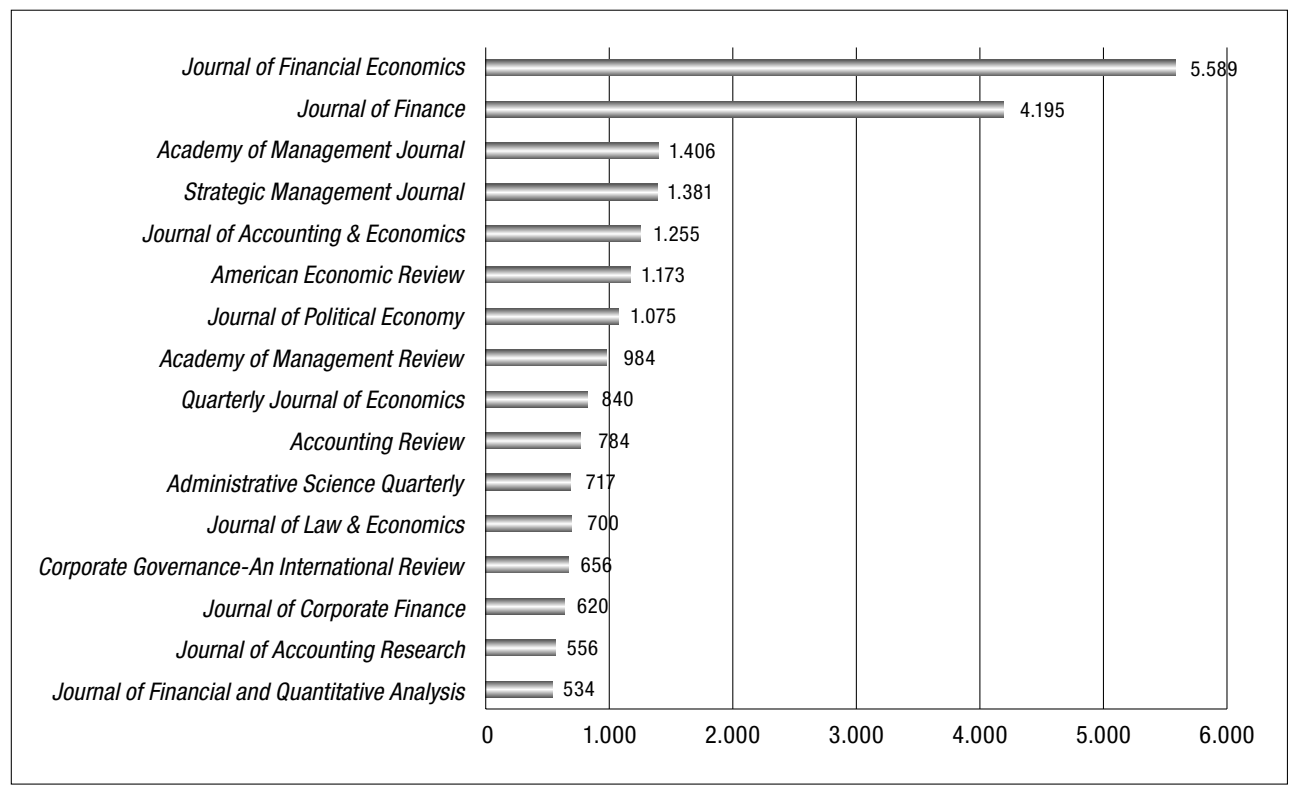

Fuente: elaboración propia a partir de los datos del ISI WoS.

\subsubsection{Revistas donde más se publica}

Las revistas científicas a las que más han recurrido los investigadores para publicar sus resultados se encuentran detalladas en la tabla V. Las quince revistas que aparecen en esta tabla recogen el 41,61\% de los artículos publicados.

Entre las revistas que los autores han escogido en mayor porcentaje para publicar los resultados de sus investigaciones se encuentra en primer lugar Corporate Governance: An International Review, surgida en 1993, como la revista de referencia sobre gobierno corporativo. En segunda posición del ranking de revistas se encuentra el Journal of Financial Economics, seguido del Journal of Business Ethics y el Journal of Corporate Finance. Parece lógico que el mayor número de artículos (192 publicaciones) se encuentre recogido en una revista especializada como es Corporate Governance: An International Review, pero también resulta ilustrativo de que 393 publicaciones de las que se muestran en la tabla $\mathrm{V}$ se encuentran recogidas en revistas especializadas en finanzas. Este hecho da a entender una vez más que la investigación sobre gobierno corporativo se realiza predominantemente desde un enfoque financiero y en menor medida desde la ética (103 publicaciones) o el derecho (55 publicaciones) y, también, que las revistas de referencia en finanzas también lo son para publicar los resultados de la investigación en gobierno corporativo. 


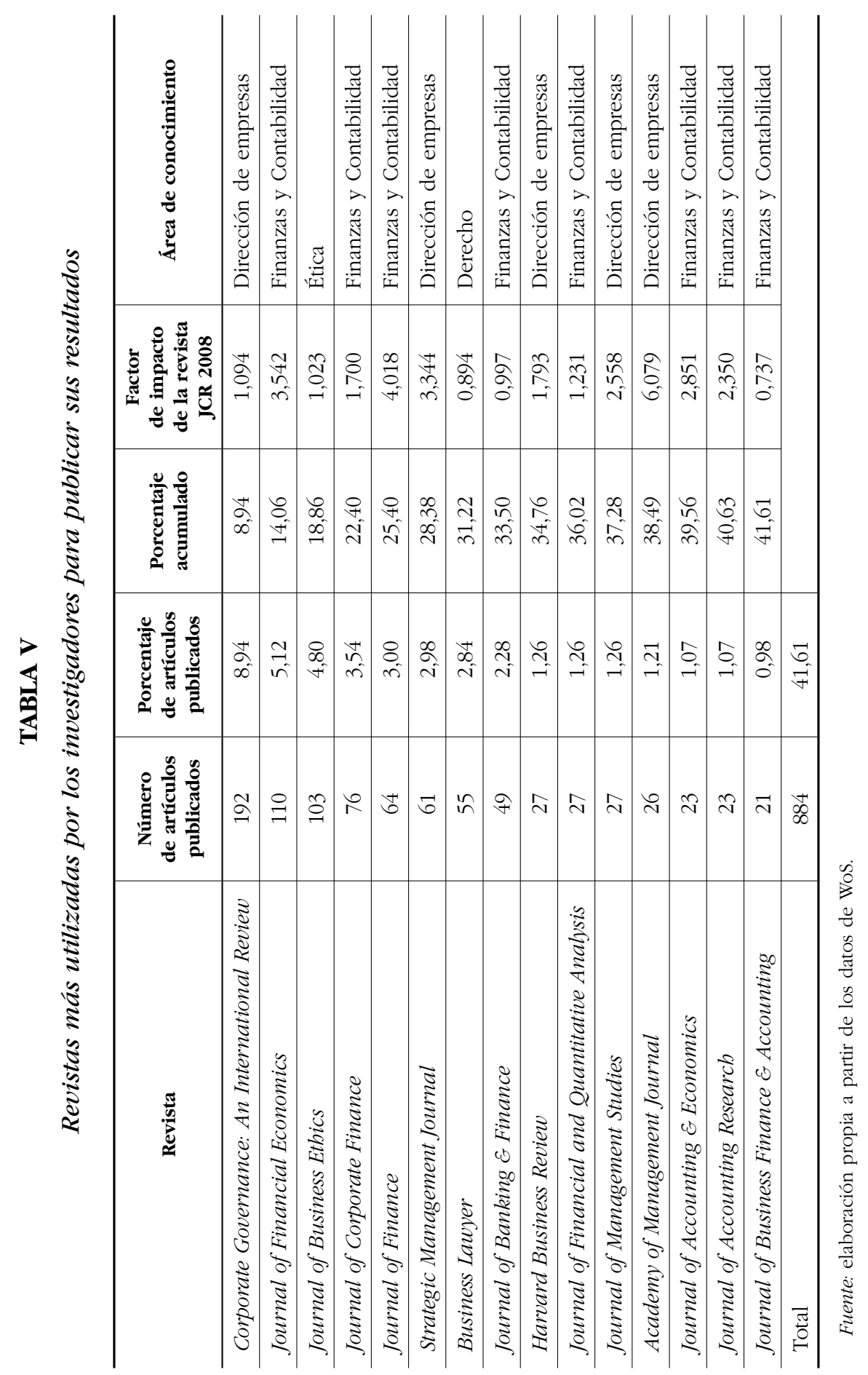


Se ha extendido este análisis mediante la aplicación de la Ley de Bradford o Ley de la dispersión de la literatura cientifica según la cual si se disponen las revistas científicas de acuerdo con la producción decreciente de artículos sobre un tema, pueden dividirse en un núcleo de publicaciones más especialmente dedicadas al tema y en varias zonas que contienen cada una de ellas el mismo número de artículos en progresión geométrica del tipo 1:n: $n^{2} \ldots$ (López, 1996). En nuestro caso, el número total de revistas entre las que se distribuyen los 2.147 artículos es de 321 y siguiendo el modelo de Bradford, éstas se han ordenado de forma decreciente en función de los artículos publicados. Se ha escogido Corporate Governance: An International Review como núcleo dada la especificidad de la revista y el destacado número de artículos publicados. Esta elección ha determinado la magnitud aproximada de cada una de las zonas (alrededor de 200 publicaciones) lo que a su vez ha determinado 9 zonas de distribución de artículos. Los resultados obtenidos se pueden consultar en la tabla VI. Para ilustrar la dispersión de las revistas, utilizando como ejemplo el caso más extremo, tendríamos que vaciar el contenido de 156 revistas (las pertenecientes a la zona 9) para tener acceso a un número parecido de artículo que si utilizáramos como única fuente de información Corporate Governance: An International Review (núcleo).

\section{TABLA VI}

\section{Productividad de las revistas}

\begin{tabular}{l|c|c|c|c|c|c}
\hline & $\begin{array}{c}\text { Número } \\
\text { de revistas }\end{array}$ & $\begin{array}{c}\text { Número } \\
\text { de artículos }\end{array}$ & $\begin{array}{c}\text { Porcentaje } \\
\text { de revistas } \\
\text { por zona }\end{array}$ & $\begin{array}{c}\text { Porcentaje } \\
\text { acumulado } \\
\text { de revistas }\end{array}$ & $\begin{array}{c}\text { Porcentaje } \\
\text { de artículos } \\
\text { por zona }\end{array}$ & $\begin{array}{c}\text { Porcentaje } \\
\text { acumulado } \\
\text { de artículos }\end{array}$ \\
\hline Núcleo & 1 & 192 & 0,31 & 0,31 & 8,94 & 8,94 \\
\hline Zona 1 & 2 & 213 & 0,62 & 0,93 & 9,92 & 18,86 \\
\hline Zona 2 & 3 & 201 & 0,93 & 1,87 & 9,36 & 28,23 \\
\hline Zona 3 & 6 & 211 & 1,87 & 3,74 & 9,83 & 38,05 \\
\hline Zona 4 & 13 & 240 & 4,05 & 7,79 & 11,18 & 49,23 \\
\hline Zona 5 & 15 & 204 & 4,67 & 12,46 & 9,50 & 58,73 \\
\hline Zona 6 & 28 & 251 & 8,72 & 21,18 & 11,69 & 70,42 \\
\hline Zona 7 & 37 & 223 & 11,53 & 32,71 & 10,39 & 80,81 \\
\hline Zona 8 & 60 & 203 & 18,69 & 51,40 & 9,46 & 90,27 \\
\hline Zona 9 & 156 & 209 & 48,60 & 100,00 & 9,73 & 100,00 \\
\hline
\end{tabular}

\subsection{Palabras clave}

Se ha realizado un análisis de las palabras clave contenidas en los artículos que forman la muestra de estudio con la finalidad de conocer qué aspectos, dentro del ámbito del gobierno corporativo, han concitado mayor atención por 
parte de los investigadores. Para ello se han extraído las 5.712 palabras clave que constaban en los artículos publicados desde 1990, año a partir del cual ISI WoS dispone de dicha información. Estas palabras clave fueron agrupadas por repeticiones y se analizaron las 50 primeras palabras clave con más repeticiones en los artículos que, a su vez, representan el $46 \%$ de todas las palabras clave extraídas. De esta lista de 50 palabras clave se eliminó el termino genérico corporate governance. A continuación se volvieron a agrupar las palabras clave, esta vez agrupando palabras clave con el mismo sentido, y se eliminaron aquéllas que tenían un sentido estrictamente económico perteneciente sólo de forma muy indirecta a conceptos de gobierno corporativo (p. ej. growth) o demasiado genéricas como para poder ser analizadas (p. ej. information o risk).

El resultado del estudio de las palabras clave se muestra en la figura 5 donde se encuentran representadas las 8 palabras clave más utilizadas y su evolución temporal. Los temas más analizados en los artículos están asociados a las palabras performance y ownership alternándose ambas entre la primera y la segunda posición de las palabras clave más utilizadas. Se puede considerar, en consecuencia, que estas dos palabras son las que resumen el principal interés de los investigadores en gobierno corporativo que, además, tienen una estrecha concordancia con los artículos más citados, ya que corresponden a las líneas de investigación que utilizarían como base los artículos de La Porta y otros (centrados en la estructura de propiedad que mejor protege a los accionistas, entre otros) y los artículos que aproximan el valor de las compañías con su desarrollo en gobierno

\section{FIGURA 5}

\section{Evolución temporal de las palabras clave}

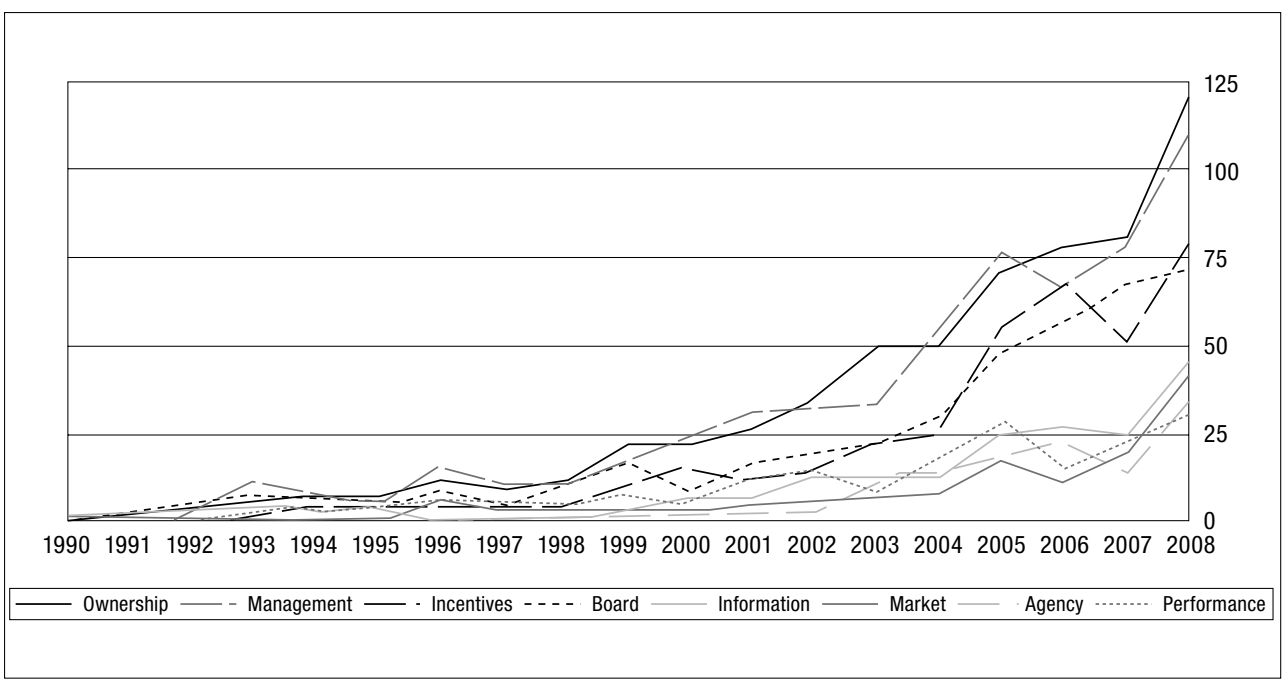

Fuente: elaboración propia a partir de los datos de ISI WoS. 
corporativo. A estas dos palabras clave más utilizadas le siguen las palabras "dirección" e "incentivos". Estas últimas podrían resumir toda aquella investigación que tenga en cuenta la dirección de la empresa por un lado y la política de incentivos de las compañías, por otro. La última ha sido de interés en todo el período estudiado pero toma especial fuerza en el año 2008, hecho que puede tener mucho que ver con los escándalos financieros y el cobro de bonus por parte de directivos durante la actual crisis financiera. Las palabras "mercado", "agencia", "información" y "consejo de administración" quedan relegadas a un segundo plano de interés científico.

\section{Conclusiones}

El gobierno corporativo constituye, desde finales de los años 90, un tema objeto de creciente interés. Este trabajo ha analizado 2.147 contribuciones científicas sobre gobierno corporativo y 79.635 referencias bibliográficas, publicadas en revistas indexadas en ISI WoS. Ningún trabajo, hasta la fecha, había contemplado un universo de análisis semejante.

El análisis realizado ha puesto de manifiesto que la investigación sobre gobierno corporativo, si bien los primeros antecedentes los encontramos en los años treinta, despega de forma importante a partir de la segunda mitad de los años noventa. Desde entonces, las publicaciones aumentan exponencialmente, siguiendo la ley de crecimiento exponencial de Price, siendo el último año del período analizado el que concentra el mayor número de contribuciones. Este resultado muestra que, en este caso, el interés académico va de la mano de la importancia del tema a nivel general e institucional, puesto que ha sido durante ese período cuando se ha promulgado la mayoría de códigos de buen gobierno empresarial. Si la proliferación de escándalos empresariales, en muchos casos ligados a la denominada crisis de las "punto com" de finales de los años noventa, significó un gran impulso a la investigación sobre gobierno corporativo, los escándalos empresariales que ha puesto de manifiesto la actual crisis financiera, y que en numerosas situaciones se ha traducido en accionistas arruinados y altos directivos enriquecidos, suponemos debería tener un impacto similar.

La investigación sobre gobierno corporativo se ajusta a la ley de Lotka, puesto que más de la mitad de los autores de las publicaciones analizadas tiene entre una y dos publicaciones y muy pocos tienen muchas publicaciones. Por otro lado, hemos observado también que los autores más citados no coinciden con los más productivos.

Respecto a las publicaciones más relevantes sobre gobierno corporativo, destaca que más de la mitad de estos trabajos se hayan publicado en las dos revistas de referencia del ámbito de finanzas como son el Journal of Finance y el Journal of Financial Economics, hecho que nos permite concluir que los fundamentos de la investigación sobre gobierno corporativo proceden, en buena medida, de la economía financiera o, al menos, la aproximación desde la 
que se investiga el fenómeno tiene una fuerte componente financiera. Este punto es importante puesto que el gobierno corporativo no es una cuestión específicamente financiera. En el total de contribuciones analizadas, destaca también la componente financiera de la investigación, puesto que más de la mitad de los trabajos se han publicado en revistas pertenecientes a este área de conocimiento.

Por último, el análisis de las palabras clave utilizadas por los autores muestra la importancia de la relación entre el gobierno corporativo y el valor de la empresa, así como las relaciones que se pueden establecer entre la estructura de propiedad de la empresa y la implementación de sistemas de incentivos apropiados que permita mejorar el gobierno corporativo.

Una extensión interesante de este trabajo será comprobar cómo afecta a la investigación en gobierno corporativo y a las conclusiones aquí presentadas, la crisis financiera internacional, oficialmente iniciada en septiembre de 2009 con la quiebra de Lehman Brothers.

\section{Bibliografía}

Ahrens, T.; Filatotchev, I., y Thomsen, S. (2009). The research frontier in corporate governance. Journal of Management and Governance, accessible en: http://www.springerlink.com/content $/ 13853457$ ?sortorder $=\mathrm{asc} \& \mathrm{v}=$ expanded\&o $=310$.

Becht, M.; Bolton, P., y Röel, A. (2003). Corporate governance and control. En: G. M. Constantinides; M. Harris, y R. M. Stulz (eds.) The Handbook of the Economics of Finance. Elsevier B.V.

Berle, A., y Means, G. (1932). The modern corporation and private property. Nueva York; Macmillan.

Bordons, M.; y Gómez-Caridad, I. (1997). La actividad científica española a través de indicadores bibliométricos en el período 1990-1993. Revista General de Información y Documentación, vol. 7 (2), 69-86.

Bordons, M.; Gómez, I.; Fernández, M. T.; Zulueta, M. A., y Méndez, Y. A. (1996). Local, domestic and international scientific collaboration in biomedical research. Scientometrics, vol. 52, 365-377.

Bordons, M., y Zulueta, M. A. (1999). Evaluación de la actividad científica a través de indicadores bibliométricos. Revista Española de Cardiología, vol. 39, 857-880.

Borokhovich, K. A.; Bricker, R. J., y Simkins, B. J. (1994). Journal communication and influence in financial research. Journal of Finance, vol. 49 (2), 713-725.

Claessens, S., y Fan, J. P. H. (2002). Corporate Governance in Asia: a survey. International Review of Finance, vol. 3 (2), 71-103.

Chan, Kam C.; Fok, Robert C. W. Pan, y Ming-Shiun (2000). Citation-based finance journal ranking: an update. Journal of Financial Practice and Education, vol. 10 (1), 132-141.

Correa, M.; García, J., y Úbeda, R. (2009). Análisis de la producción científica en Economía Financiera: 1995-2006. Revista Española de Documentación Científica, vol. 32 (2), $83-104$. 
Davis, G. (1991). Agents without principles? The spread of the poison pill through the intercorporate network. Administrative Science Quarterly, vol. 36 (4), 583-613.

Dodd, P., y Warner, J. B. (1983). On corporate governance: a study of proxy contests. Journal of Financial Economics, vol. 11 (1-4), 401-438.

Durisin, B., y Puzone, F. (2009). Maturation of Corporate Governance research: 1993-2007: An Assessment. Corporate Governance: An International Review, vol. 17 (3), 266-291.

Estes, R. M. (1977). Emerging solution to Corporate Governance. Harvard Business Review, vol. 55(6), 20.

Estrin, S., y Wright, M. (1999). Corporate Governance in the Former Soviet Union: An overview. Journal of Comparative Economics, vol. 27 (3), 398-421.

Farinha, G. (2003). Corporate Governance: a survey of the literature. Universidade do Porto Economia Discussion Paper no. 2003-06. Faculdade de Economia do Porto, University of Porto.

Filatotchev, I., y Boyd, B. (2009). Taking stock of corporate governance research while looking to the future. Corporate Governance: An International Review, vol. 17 (3), 257-265.

Gompers, P.; Ishii, J., y Metrick, A. (2003). Corporate governance and equity prices. Quarterly Journal of Economics, vol. 118 (1), 107-55.

Hart, O. (1995). Corporate Governance, some theory and applications. The Economic Journal, vol. 105, 687-689.

Instituto de Estudios Documentales sobre Ciencia y Tecnología, Centro de Ciencias Humanas y Sociales, Consejo Superior de Investigaciones Científicas (2009). La actividad científica del CSIC a través del Web of Science Estudio bibliométrico del período 20002007. Madrid; CSIC.

Jensen, M. (1993). The modern industrial revolution, exit and failure of internal control systems. Journal of Finance, vol. 48, 831-880.

Jensen, M. C., y Meckling, W. H. (1976). Theory of firm: Managerial behavior, agency costs and ownership structure. Journal of Financial Economics, vol. 3, 305-360.

Jiménez, E., y Moya, F. (2003). The evolution of research activity in Spain: The impact of the National Commision for the Evaluation of Research Activity (CNEAI). Research Policy, vol. 32, 123-142.

La Porta, R.; Lopez-de-Silanes, F., y Shleifer, A. (1999). Corporate ownership around the world. Journal of Finance, vol. 54 (2), 471-517.

La Porta, R.; Lopez-de-Silanes, F.; Shleifer, A., y Vischny, R. (1997). Legal determinants of external finance. Journal of Finance, vol. 52, 1131-1150.

La Porta, R.; Lopez-de-Silanes, F.; Shleifer, A., y Vischny, R. (1998). Law and Finance. Journal of Political Economy, vol. 106, 1113-1155.

La Porta, R.; Lopez-de-Silanes, F.; Shleifer, A., y Vischny, R. (2000). Investor protection and Corporate Governance. Journal of Financial Economics, vol. 58 (1-2), 3-27.

La Porta, R.; Lopez-de-Silanes, F.; Shleifer, A., y Vischny, R. (2002). Investor protection and corporate valuation. The Journal of Finance, vol. 57 (3), 1147-1170.

Linday, R. M.; Lindsay, L. M., y Irvine, V. B. (1996). Instilling ethical behavior in organizations: A survey of Canadian companies. Journal of Business Ethics, vol. 15, 393-407. 
López, F. J., y Pereira, M. L. (2006). Análisis internacional de los códigos de buen gobierno. Universia Business Review, vol. 11, 10-21.

López, P. (1996). Introducción a la Bibliometría. Valencia: Promolibro.

Martin, B. R., y Irvine, J. (1983). Assessing basic research: some partial indicators of scientific progress in radio astronomy. Research Policy, vol. 12, 61-90.

- Miller, M. (1997). Is American Corporate Governance fatally flawed? En: Stern, J. M., D. Chew (eds.) (2003). The Revolution in Corporate Finance. Oxford: Blackwell Publishing Oxford.

Moya, F.; Chichilla, Z.; Corera, E.; Gómez-Crisóstomo, M. R.; Gómez-Molina, A.; Muñoz F. J., y Vargas, B. (2005). Indicadores bibliométricos de la actividad científica española. Madrid: Fecyt.

Porter, M. (1997). Capital choices: changing the way America invests in industry. En: Chew, D. H. (eds.). Studies in International Corporate Finance and Governance Systems-A comparison of the US, Japan and Europe. Nueva York: Oxford University Press.

Prigge, S. (1998). A survey of German Corporate Governance. Oxford; Clarendon Press.

Reyes, M. J.; Guerrero, V. P., y Moya, F. (2006). Proyección internacional de la investigación en Extremadura. Revista Española de Documentación Científica, vol. 24 (4), 525550.

Shleifer, A., y Vishny, R. W. (1997). A survey of corporate governance. Journal of Finance, vol. 52, 737-783.

Walsh, P. P., y Whelan, C. (2001). Firm performance and the political economy of corporate governance: survey evidence for Bulgaria, Hungary, Slovakia and Slovenia. Economic Systems, vol. 25(2), 85-112.

Williamson, O. E. (1988). Corporate Finance and Corporate Governance. The Journal of Finance, vol. 43 (3), 567-591.

Yermack, D. (1996). Higher market valuation of companies with a small board of directors. Journal of Financial Economics, vol. 40 (2), 185- 211.

Zingales, L. (1998). Corporate Governance. En: Newman, P. (eds.). The new Palgrave Dictionary of Economics and the Law. Londres: Macmillan. 